\title{
Transcriptome network analysis reveals potential candidate genes for squamous lung cancer
}

\author{
$\mathrm{JING} \mathrm{BAI}^{1}$ and SHENG HU ${ }^{2}$ \\ ${ }^{1}$ Department of Cardiology, Renmin Hospital of Wuhan University; ${ }^{2}$ Department of \\ Medical Oncology, Hubei Cancer Hospital, Wuhan 430060, P.R. China
}

Received June 14, 2011; Accepted August 3, 2011

DOI: $10.3892 / \mathrm{ijmm} .2011 .796$

\begin{abstract}
Squamous lung cancer is a common type of lung cancer; however, its mechanism of oncogenesis is still unknown. The aim of this study was to screen candidate genes of squamous lung cancer using a bioinformatics strategy and elucidate the mechanism of squamous lung cancer. Published microarray data of the GSE3268 series was obtained from Gene Expression Omnibus (GEO). Significance analysis of microarrays was performed using the software $\mathrm{R}$, and differentially expressed genes by $\mathrm{R}$ analysis were harvested. The relationship between transcription factors and target genes in cancer were collected from the Transcriptional regulatory element database. A transcriptome network analysis method was used to construct gene regulation networks and select the candidate genes for squamous lung cancer. SPI1, FLI1, FOS, ETS2, EGR1 and PPARG were defined as candidate genes for squamous lung cancer by the transcriptome network analysis method. Among them, 5 genes had been reported to be involved in lung cancer, except SPI1 and FLI1. Effective recall on previous knowledge conferred strong confidence in these methods. It is demonstrated that transcriptome network analysis is useful in the identification of candidate genes in disease.
\end{abstract}

\section{Introduction}

Lung cancer is the most common cause of cancer deaths worldwide. Every year, 1.3 million people die from this disease, which is mainly caused by smoking. It is projected that by 2020, lung cancer will be the fifth most lethal entity among all diseases (1). Improvement in survival has been very modest. Less than $16 \%$ of lung cancer patients survive 5 years or more, owing to late diagnosis and a paucity of effective therapies.

Correspondence to: Dr Jing Bai, Department of Cardiology, Renmin Hospital of Wuhan University, Wuhan 430060, P.R. China

E-mail: jingbaidoc@gmail.com

Key words: squamous lung cancer, transcription factor, transcriptome network analysis
Squamous cell carcinoma (SqCC) and adenocarcinoma (AC) are the predominant non-small cell lung cancer (NSCLC) cell types. If NSCLC is diagnosed before it has spread from the lungs (stage I disease), it can be surgically removed and many patients with stage I NSCLC survive for more than 5 years after their diagnosis. Unfortunately, in more than half of patients, NSCLC has metastasized before it is diagnosed. Surgery remains the best treatment for a curative outcome of NSCLC $(2,3)$.

Efforts of intensive research to increase our understanding of the molecular basis of lung cancer have been undertaken. Research proved that the cancer cell has been characterized by several genetic changes that lead to altered cellular functions and multiple factors of the cancer-cell environment further affect the tumor cell via various receptors and subsequent signaling pathways (4). The molecular mechanisms of carcinogenesis in lung cancer are complex and involve multiple oncogenes, tumor suppressor genes, receptor tyrosine kinases, cytoplasmic enzymes, and tumor interstitial elements, among other cellular proteins (5). Hedgehog, Notch and Wnt signaling, which are essential for axial patterning and progenitor cell fates in signalling pathways conserved from flies to humans, participate in lung development. A deeper understanding of the these pathways responsible for airway epithelial repair and tumorigenesis, such as Hedgehog (Hh), Notch, and Wingless (Wnt), can lead to the development of newer and more specific therapies for lung cancer (6). Research also showed that the majority of non-small cell lung cancer (NSCLC) exhibits dysregulated antiapoptotic pathways involving the transcription factor $\mathrm{NF}-\kappa \mathrm{B}(7,8)$. There is growing evidence showing that transcription factors play a critical role in the invasion and metastasis of tumors by regulating transcription and expression of many metastasis associated genes.

An understanding of these new developments in transcription factors and pathways may pave the way for innovative combinatorial approaches for treatment of lung cancer and possibly chemoprevention. However, most of previous studied on transcription were primarily on protein and mRNA expression, and high-throughput functional analysis of multiple transcription factors in human squamous lung cancer is rare. In this study, we aimed to identify potential candidate genes associated with squamous lung cancer by transcriptional network analysis and explored new predictive biomarkers and targets genes. 


\section{Materials and methods}

\section{Data source}

Affymetrix microarray data. One transcription profiles of squamous lung cancer GSE3268 were obtained from a public functional genomics data repository GEO (http://www.ncbi. nlm.nih.gov/geo/) which are based on the Affymetrix GPL96 platform data (Affymetrix Human Genome U133A Array) (9). Each pair of samples represents a single patient with squamous lung cancer. One is derived from the cancer cells, and the other is from normal cells.

Pathway data. KEGG (Kyoto Encyclopedia of Genes and Genomes) is a collection of online databases dealing with genomes, enzymatic pathways, and biological chemicals (10). The PATHWAY database records networks of molecular interactions in the cells, and variants of them specific to particular organisms (http://www.genome.jp/kegg/). Total 130 pathways, involving 2287 genes, were collected from KEGG.

Regulation data. There are 2600 proteins in the human genome that contain DNA-binding domains, and most of these are presumed to function as transcription factors (9). The combinatorial use of a subset of the 2000 human transcription factors easily accounts for the unique regulation of each gene in the human genome during development (11).

These transcription factors are grouped into 5 super class families, based on the presence of conserved DNA-binding domains. TRANSFAC database contains data on transcription factors, their experimentally-proven binding sites, and regulated genes (12).

Transcriptional regulatory element database (TRED) has been built in response to increasing needs of an integrated repository for both cis- and trans-regulatory elements in mammals (13). TRED performed the curation for transcriptional regulation information, including transcription factor binding motifs and experimental evidence. The curation is currently focusing on target genes of 36 cancer-related TF families.

Pairs (774) of regulatory relationship between 219 transcription factors (TFs) and 265 target genes were collected from TRANSFAC (http://www.gene-regulation.com/pub/ databases.html.

Pairs (5722) of regulatory relationship between 102 transcription factors (TFs) and 2920 target genes were collected from TRED (http://rulai.cshl.edu/TRED/).

Combined the two regulation datasets, total 6328 regulatory relationships between 276 TFs and 3002 target genes were collected (Table I).

\section{Methods}

Differentially expressed gene (DEG) analysis. For the GSE3268 dataset, the limma method (14) was used to identify DEGs. The original expression datasets from all conditions were processed into expression estimates using the RMA method with the default settings implemented in Bioconductor, and then the linear model was constructed. The DEGs with the fold change value $>2$ were selected.
Co-expression analysis. For demonstrating the potential regulatory relationship, the Pearson correlation coefficient (PCC) was calculated for all pair-wise comparisons of geneexpression values between TFs and the DEGs. The regulatory relationships whose absolute PCC are $>0.6$ were considered as significant.

Gene ontology analysis. The BiNGO analysis (15) was used to identify over-represented GO categories in biological process. The result of Gene Ontology analysis was shown in Table II.

Regulation network construction. Using the regulation data that have been collected from TRANSFAC database and TRED database, we matched the relationships between differentially expressed TFs and its differentially expressed target genes.

Based on the above two regulation datasets and the pathway relationships of the target genes, we build the regulation networks by Cytoscape (16). Based on the significant relationships (PCC $>0.6$ or PCC $<-0.6$ ) between TFs and its target genes, 57 putative regulatory relationships were predicted between 12 TFs and 45 target genes. The results of regulation network are shown in Fig. 1.

Significance analysis of pathway. We adopted an impact analysis that includes the statistical significance of the set of pathway genes but also considers other crucial factors such as the magnitude of the expression change of each gene, the topology of the signaling pathway, their interactions and so on (17). In this model, the impact factor (IF) of a pathway Pi is calculated as the sum of two terms:

$$
I F(P i)=\log \left(\frac{1}{p i}\right)+\frac{\sum_{g \in P i}|P F(g)|}{|\Delta E| \cdot N_{d c}(P i)}
$$

The first term is a probabilistic term that captures the significance of the given pathway $P_{i}$ from the perspective of the set of genes contained in it.

It is obtained by using the hyper geometric model in which $p_{i}$ is the probability of obtaining at least the observed number of differentially expressed gene, $N_{d e}$, just by chance $(18,19)$.

The second term is a functional term that depends on the identity of the specific genes that are differentially expressed as well as on the interactions described by the pathway (i.e., its topology).

The second term sums up the absolute values of the perturbation factors (PFs) for all genes $g$ on the given pathway $P_{i}$. The PF of a gene $g$ is calculated as follows:

$$
P F(g)=\Delta E(g)+\sum_{u \in U S g} \beta_{\text {gg }} \cdot \frac{P F(u)}{N_{d s}(u)}
$$

In this equation, the first term $\Delta E(g)$ captures the quantitative information measured in the gene expression experiment. The factor $\Delta E(g)$ represents the normalized measured expression change of the gene $g$. The first term $\Delta E(g)$ in the above equation is a sum of all PFs of the genes $u$ directly upstream of the target gene $g$, normalized by the number of downstream genes of each such gene $N_{d s}(u)$, and weighted by a factor $\beta_{u g}$, which reflects the type of interaction: $\beta_{u g}=1$ for induction, 
Table I. Regulation data form TRANSFAC and TRED.

\begin{tabular}{lcccc}
\hline Source & Regulation & TFs & Targets & Links \\
\hline TRANSFAC & 774 & 219 & 265 & http://www.gene-regulation.com/pub/databases.html \\
TRED & 5722 & 102 & 2920 & http://rulai.cshl.edu/TRED/ \\
Total & 6328 & 276 & 3002 & \\
\hline
\end{tabular}

Table II. Go enrichments analysis.

\begin{tabular}{llr}
\hline GO-ID & \multicolumn{1}{c}{ Description } & p-value \\
\hline 48518 & Positive regulation of biological process & $2.22 \mathrm{E}-11$ \\
48522 & Positive regulation of cellular process & $8.67 \mathrm{E}-11$ \\
42221 & Response to chemical stimulus & $1.56 \mathrm{E}-10$ \\
6950 & Response to stress & $2.11 \mathrm{E}-10$ \\
48513 & Organ development & $2.71 \mathrm{E}-10$ \\
50896 & Response to stimulus & $4.36 \mathrm{E}-10$ \\
51239 & Regulation of multicellular organismal process \\
2376 & Immune system process & $9.64 \mathrm{E}-10$ \\
48731 & System development & $9.74 \mathrm{E}-10$ \\
7275 & Multicellular organismal development & $7.33 \mathrm{E}-09$ \\
\hline
\end{tabular}

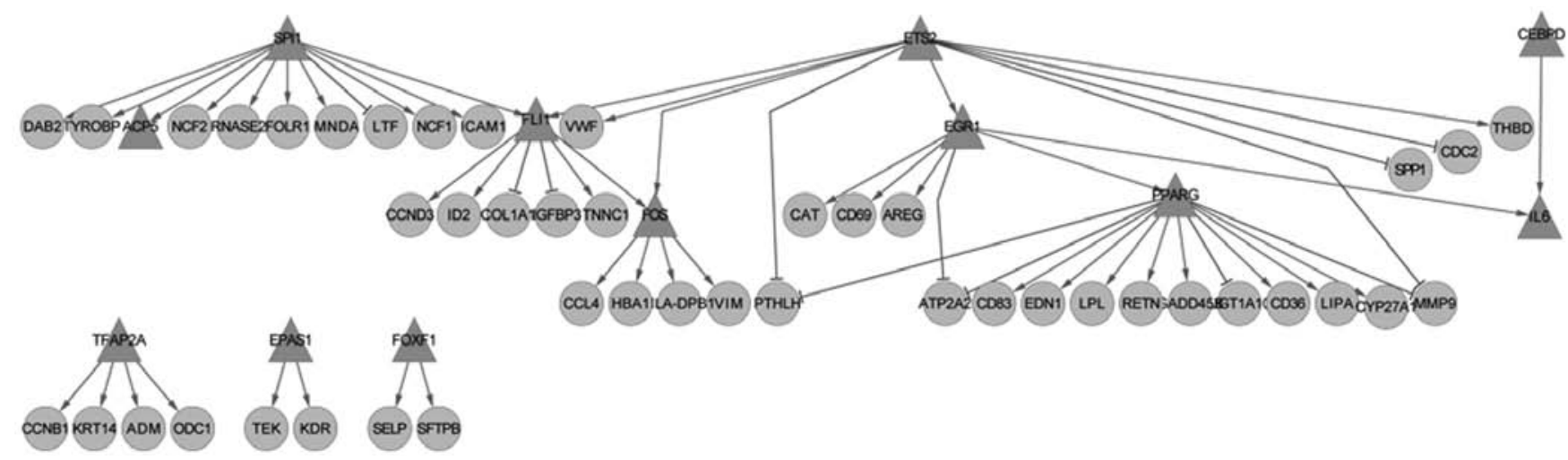

Figure 1. Regulation network construction in squamous lung cancer. The triangle denotes transcription factor and the circle denotes targeting genes. The arrow line suggests that the transcription factor activates their target gene in squamous lung cancer. In contrast, the other type of line suggests that the transcription factor inhibits the expression of their target gene in squamous lung cancer.

$\beta_{u g}=-1$ for repression (KEGG supply this information on the type of interaction of two genes in the description of the pathway topology). $U S_{g}$ is the set of all such genes upstream of $g$. We need to normalize with respect to the size of the pathway by dividing the total perturbation by the number of differentially expressed genes on the given pathway, $N_{d e}\left(P_{i}\right)$. In order to make the $I F \mathrm{~s}$ as independent as possible from the technology, and also comparable between problems, we also divide the second term in equation 1 by the mean absolute fold change $\Delta E$, calculated across all differentially expressed genes. The result of the significance analysis of pathway is shown in Fig. 2.

Regulation network between TFs and pathways. To further investigate the regulatory relationships between TFs and path- ways, we mapped DEGs to pathways and gained a regulation network between TFs and pathways.

\section{Results}

Regulation network construction in squamous lung cancer. To get pathway-related DEGs of squamous lung cancer, we obtained publicly available microarray data sets GSE3268 from GEO. After microarray analysis, the differentially expressed genes with the fold change value $>2$ of GSE3268 were selected. Genes (1298) were selected as DEGs from GSE3268. To get the regulatory relationships, the co-expressed value ( $\mathrm{PCC} \geq 0.6$ ) was chosen as the threshold. Finally, we got 57 regulatory relationships between 12 differently expressed TFs and their 45 differently expressed target genes. By 


\begin{tabular}{|c|c|c|c|c|c|}
\hline \multirow{2}{*}{$\begin{array}{l}\text { Input Genes I } \\
\text { Pathway Genes }\end{array}$} & \multicolumn{2}{|c|}{ Hypergeometric } & \multicolumn{2}{|c|}{ If Analysis p-value } & \multirow[b]{2}{*}{ Impact Factor } \\
\hline & Raw & Flag & Raw & Corrected & \\
\hline $14 / 119$ & $5.179 E-2$ & & $0.000 E 0$ & $0.000 \mathrm{E} 0$ & 221.519 \\
\hline $24 / 134$ & $2.682 E-5$ & & $0.000 E 0$ & $0.000 \mathrm{E} 0$ & 116.444 \\
\hline $10 / 89$ & $1.124 \mathrm{E} \cdot 1$ & & $0.000 E 0$ & $0.000 E 0$ & 66.177 \\
\hline $10 / 78$ & $7.474 \mathrm{E} \cdot 2$ & & $1.120 E \cdot 10$ & $1.120 \mathrm{E} \cdot 10$ & 26.216 \\
\hline $17 / 69$ & $1.662 E-5$ & & $2.665 E-5$ & $2.665 \mathrm{E}-5$ & 13.185 \\
\hline $23 / 118$ & $1.108 E \cdot 5$ & & $4.781 \mathrm{E}-5$ & $4.781 \mathrm{E} \cdot 5$ & 12.555 \\
\hline $6 / 76$ & $5.345 E \cdot 1$ & & $6.652 E \cdot 5$ & $6.652 \mathrm{E} \cdot 5$ & 12.198 \\
\hline $18 / 84$ & $8.014 E-5$ & & $1.937 E-4$ & $1.937 \mathrm{E}-4$ & 11.037 \\
\hline $21 / 144$ & $1.432 \mathrm{E}-4$ & & $6.702 E-4$ & $6.702 \mathrm{E}-4$ & 9.676 \\
\hline $10 / 36$ & $2.172 E \cdot 4$ & & $9.058 \mathrm{E} \cdot 4$ & $9.058 \mathrm{E} \cdot 4$ & 9.343 \\
\hline $29 / 203$ & $1.004 \mathrm{E} \cdot 3$ & & $9.058 \mathrm{E}-4$ & $9.058 \mathrm{E}-4$ & 9.343 \\
\hline $14 / 69$ & $4.080 \mathrm{E} \cdot 4$ & & $1.137 \mathrm{E} \cdot 3$ & $1.137 \mathrm{E} \cdot 3$ & 9.091 \\
\hline $43 / 330$ & $5.779 E-4$ & & $1.262 E-3$ & $1.262 \mathrm{E} \cdot 3$ & 8.975 \\
\hline $13 / 70$ & $1.358 \mathrm{E} \cdot 3$ & & $3.680 \mathrm{E} \cdot 3$ & $3.680 \mathrm{E} \cdot 3$ & 7.777 \\
\hline $9 / 42$ & $1.325 E \cdot 3$ & $?$ & $4.236 E-3$ & $4.236 E-3$ & 7.618 \\
\hline $15 / 102$ & $7.041 \mathrm{E}-3$ & & $9.831 E-3$ & $9.831 E-3$ & 6.658 \\
\hline $9 / 44$ & $3.057 \mathrm{E} \cdot 3$ & & $1.013 E \cdot 2$ & $1.013 E \cdot 2$ & 6.623 \\
\hline $9 / 55$ & $1.143 \mathrm{E} \cdot 2$ & $?$ & $1.035 E \cdot 2$ & $1.035 \mathrm{E} \cdot 2$ & 6.599 \\
\hline $14 / 84$ & $5.933 E-3$ & ? & $1.160 \mathrm{E}-2$ & $1.160 \mathrm{E} \cdot 2$ & 6.467 \\
\hline $8 / 38$ & $4.412 E-3$ & $?$ & $1.397 \mathrm{E}-2$ & $1.397 \mathrm{E}-2$ & 6.252 \\
\hline $13 / 135$ & $1.789 \mathrm{E}-1$ & & $2.950 E-2$ & $2.950 \mathrm{E} \cdot 2$ & 5.376 \\
\hline $6 / 30$ & $2.016 \mathrm{E}-2$ & $?$ & $5.288 \mathrm{E}-2$ & $5.288 \mathrm{E}-2$ & 4.676 \\
\hline $7 / 42$ & $4.775 \mathrm{E} \cdot 2$ & $?$ & $5.620 \mathrm{E}-2$ & $5.620 \mathrm{E} \cdot 2$ & 4.602 \\
\hline $14 / 129$ & 7.963E-2 & & $6.380 \mathrm{E}-2$ & $6.380 \mathrm{E}-2$ & 4.447 \\
\hline $16 / 152$ & $8.343 \mathrm{E} \cdot 2$ & & $6.603 E \cdot 2$ & $6.603 \mathrm{E} \cdot 2$ & 4.405 \\
\hline $10 / 69$ & $5.470 \mathrm{E}-2$ & $?$ & $7.339 E-2$ & $7.339 \mathrm{E} \cdot 2$ & 4.275 \\
\hline $11 / 87$ & $7.189 \mathrm{E}-2$ & & $7.537 E-2$ & $7.537 \mathrm{E}-2$ & 4.242 \\
\hline $26 / 272$ & $1.188 \mathrm{E} \cdot 1$ & & $8.224 E \cdot 2$ & $8.224 \mathrm{E} \cdot 2$ & 4.134 \\
\hline $8 / 59$ & $8.606 \mathrm{E}-2$ & 2 & $9.320 E-2$ & $9.320 \mathrm{E}-2$ & 3.978 \\
\hline $8 / 53$ & $4.495 \mathrm{E}-2$ & 2 & $9.677 \mathrm{E}-2$ & $9.677 \mathrm{E}-2$ & 3.931 \\
\hline $13 / 108$ & $8.418 \mathrm{E} \cdot 2$ & 2 & $1.064 \mathrm{E}-1$ & $1.064 \mathrm{E}-1$ & 3.812 \\
\hline $15 / 138$ & $1.138 \mathrm{E} \cdot 1$ & & $1.113 \mathrm{E} \cdot 1$ & $1.113 \mathrm{E} \cdot 1$ & 3.755 \\
\hline $9 / 87$ & $1.996 \mathrm{E}-1$ & & $1.409 \mathrm{E}-1$ & $1.409 E-1$ & 3.453 \\
\hline $8 / 96$ & 4.763E-1 & & $1.423 E-1$ & $1.423 \mathrm{E}-1$ & 3.441 \\
\hline $4 / 75$ & $8.538 \mathrm{E} \cdot 1$ & 2 & $1.491 \mathrm{E}-1$ & $1.491 \mathrm{E}-1$ & 3.38 \\
\hline
\end{tabular}

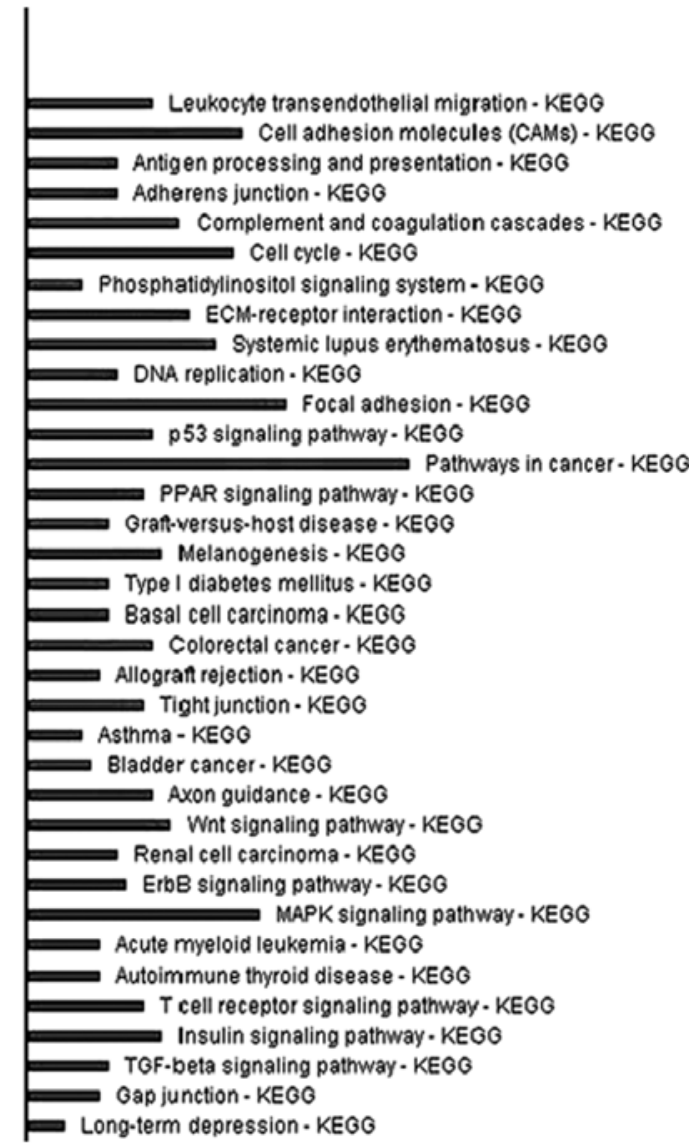

Figure 2. Significance analysis of pathway in squamous lung cancer. Impact factor (IF) score denotes the significance of the pathway in squamous lung cancer. A higher impact factor score suggests that this pathway is more significant in squamous lung cancer, and vice versa.

integrating the regulatory relationships above, a regulation network of squamous lung cancer was build between TFs and its target genes (Fig. 1). In this network, SPI1, FLI1, FOS, ETS2, EGR1 and PPARG with a higher degree form a local network suggesting that these genes may play an important role in squamous lung cancer. Besides, transcription factor cascade Ets2->egr1-->pparg-->target genes and ets2 and sp1 ->fli1-->fos$\rightarrow$ target genes was also observed in this network.

GO analysis of the regulation network in squamous lung cancer. Several Gene Ontology (GO) categories were enriched among these genes in the regulatory network, including positive regulation of the biological process, positive regulation of the cellular process, response to chemical stimulus and response to stress (Table II).

Significant pathway in squamous lung cancer. To identify the relevant pathways changed in squamous lung cancer, we used a statistical approach at pathway level. Significance analysis at single gene level may suffer from the limited number of samples and experimental noise that can severely limit the power of the chosen statistical test. Pathway can provide an alternative way to relax the significance threshold applied to single genes and may lead to a better biological interpretation. Therefore, we adopted a pathway-based impact analysis method that contained many factors including the statistical significance of the set of differentially expressed genes in the pathway, the magnitude of the expression change of each gene, the topology of the signaling pathway, their interactions and so on. The impact analysis method yields many significant pathways such as leukocyte transendothelial migration, cell adhesion molecules (CAMs), antigen processing and presentation, adherens junction, complement and coagulation cascades, cell cycle, phosphatidylinositol signaling system, ECM-receptor interaction, systemic lupus erythematosus, DNA replication, focal adhesion, p53 signaling pathway, pathways in cancer, PPAR signaling pathway (Fig. 2).

Regulation network between TFs and pathways in squamous lung cancer. To further investigate the regulatory relationships between TFs and pathways, we mapped DEGs to pathways and got a regulation network between TFs and pathways (Fig. 3). In the network, SPI1, FLI1, FOS, ETS2, EGR1 and PPARG were shown as a hub linked to many lung cancer pathways.

\section{Discussion}

Much of the biological diversity of tumors is the result of variation in the transcriptional programs (20). Transcriptional profiling of cancer using microarray has revolutionized the field by allowing researchers to discover tumor subclasses and target genes for diagnosis and therapy.

The interpretation of the genes identified by the microarray analysis was analyzed in a context where a single gene was 


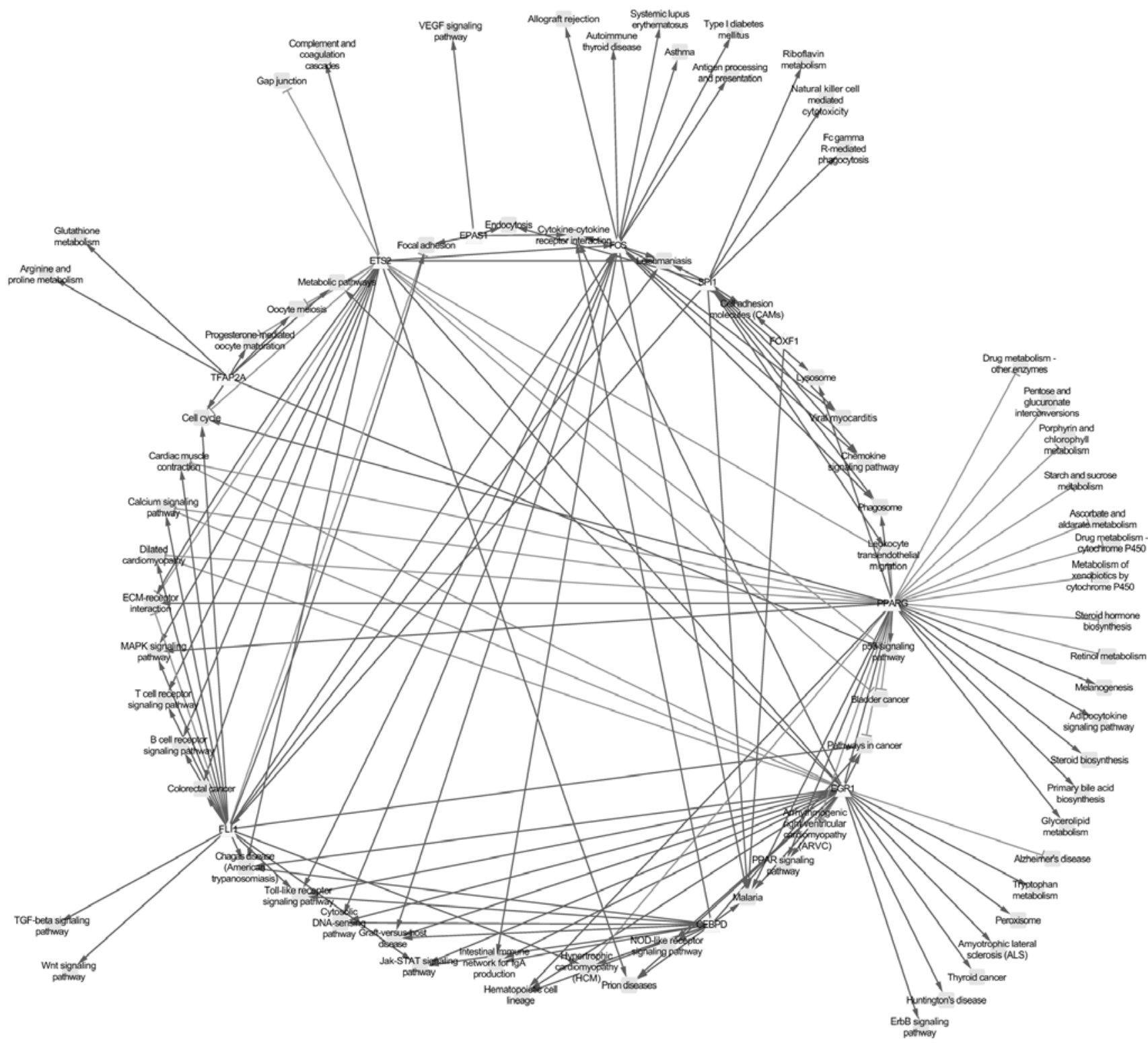

Figure 3. Regulation network between TFs and pathways in squamous lung cancer. The triangle denotes a transcription factor and diamonds denotes a pathway. The arrow line suggests that the transcription factor activates the pathways in squamous lung cancer. In contrast, the other type of line suggests that the transcription factor inhibits the expression of the pathways in squamous lung cancer.

accounted for the individual phenotype. However, this ignores the fact that proteins rarely act in isolation from one another, and their specific functions are determined by association with other proteins. Systems biology, is driven by the gradual realization that a single gene is seldom accountable for a discrete biological function (21). In response, contemporary biology has amassed a battery of methods to survey the global features of the cells, from DNA, RNA and proteins to small molecules $(22,23)$.

Network and pathway analysis can be used to interrogate gene expression data in order to understand the biological processes affected by a particular manipulation or disease/ condition of interest.

A systems-level understanding of the biological processes affected in particular disease states can allow the identification of candidates not only for pharmaceutical intervention but also for potential prognostic and diagnostic markers for the disease.
Many transcription factors are involved in the progress of squamous lung cancer. Some of the transcription factors and its target genes take part in the progress in squamous lung cancer. Gene regulation networks of squamous lung cancer were constructed to explain the relationship between the transcription factor and pathway in squamous lung cancer by a systems biology method.

The regulation network shows that the transcription factor WRKY53 plays a key role in the regulatory network through regulating ATWRKY18 and GBF3.

The transcriptome network analysis using a transcription factor and target gene relationship suggest that the genes up-regulated in squamous lung carcinomas are rich in hubs. These hubs include SPI1, FLI1, FOS, ETS2, EGR1, and PPARG.

PPARG, also known as PPAR- $\gamma$, encodes a member of the peroxisome proliferator-activated receptor (PPAR) subfamily of nuclear receptors. (PPAR)- $\gamma$ belongs to the nuclear hormone 
receptor superfamily of ligand-dependent transcription factors. It is a mediator of adipocyte differentiation, regulates lipid metabolism and macrophage function. Activation of PPAR- $\gamma$ was shown to modulate various hallmarks of cancer through its pleiotropic affects on multiple different cell types in the tumor microenvironment. An overwhelming number of preclinical-studies demonstrate the efficacy of PPAR- $\gamma$ ligands in the control of tumor progression through their affects on various cellular processes, including cell proliferation, apoptosis, angiogenesis, inflammation and metastasis.

Studies demonstrated that ligands of PPAR- $\gamma$ induce differentiation of lung cancer cells and inhibit their growth (24). Overexpression of PPAR- $\gamma$ cDNA alone was sufficient to inhibit tumor growth in vivo, cellular migration and invasion in vitro in lung cancer cells (25). Researches also suggest that by inhibiting fibronectin and its receptor, PPAR- $\gamma$ ligands disrupt tumor cell and ECM interactions essential for tumor cell proliferation and PPAR- $\gamma$ ligands inhibit $\alpha 5$ integrin gene transcription in non-small cell lung carcinoma cells. Findings also indicate that the reduction in 15-LOX, 15(S)-HETE and 13(S)-HODE results in the decreased PPAR- $\gamma$ activity seen in lung tumours and contributes to the development of lung tumours induced by tobacco smoking (26). Research found that PPAR- $\gamma$ inhibits transformed growth of non-small cell lung cancer cells through selective suppression of Snail (27). Research suggest that the enhancement of PPAR- $\gamma$ activity with its ligands, and the suppression of PPAR- $\alpha$ with its inhibitors, may prevent the formation of lung tumors, as well as accelerate the therapy of lung cancer (28). Also, the relationship between PPAR- $\gamma$ cross-regulation with other signaling pathways and lung cancer therapy have attracted research attention (29).

It has been reported that EGR1 is expressed in human lung cancer and in normal lung tissue (30). Western blotting revealed that polyethylene combustion products also upregulated EGR1 (31). Results suggest that siRNA-Egr-1 alone or in combination with camptothecin could be a potent antineoplastic agent in suppressing the growth of breast tumor (32). Downregulation of stathmin expression is mediated directly by EGR1 and associated with p53 activity in the lung cancer cell line A549 (33). It was found that EGR1 is a novel regulator of stathmin expression and $\mathrm{p} 53$ mediates the transcriptional repression of stathmin by EGR1 in human lung cancer cells. Data suggest that EGR1 plays important roles on VEGF-A expression in lung cancer cells, and epigenetic silencing of transactivator(s) associated with NAB-2 expression might also contribute to upregulation of VEGF-A expression. Recent findings suggest that EGR1 plays important roles on VEGF-A expression in lung cancer cells, and epigenetic silencing of transactivator(s) associated with NAB-2 expression might also contribute to upregulation of VEGF-A expression (34). In a genome-wide association study, rs1209950 in the ETS2 gene were identified as new prognostic biomarker candidates for advanced NSCLC treated with CBDCA and PTX (35).

The FOS proteins have been implicated as regulators of cell proliferation, differentiation and transformation. In some cases, expression of the FOS gene has also been associated with apoptotic cell death. Interleukin-7/interleukin-7 receptor induce c-Fos/c-Jun-dependent vascular endothelial growth factor-D up-regulation: a mechanism of lymphangiogenesis in lung cancer. Our results provide evidence that IL-7/IL-7R induce VEGF-D up-regulation and promote lymphangio-genesis via c-Fos/c-Jun pathway in lung cancer (36). Vasoactive intestinal peptide can increase the expression and secretion of VEGF in lung cancer cells by activating the transcription factor c-fos, then promote the angiogenesis of lung cancer and thus play an important role in the pathogenesis of lung cancer (37).

Effective recall on previous knowledge conferred us strong confidence in these methods. It is demonstrated that transcriptome network analysis is useful in identification of the candidate genes in squamous lung cancer. However, to the best of our knowledge, there is no study on the relationship between SPI1 and lung cancer, or SPI1 and FLI1 also appearing as hubs. SPI1 gene encodes an ETS-domain transcription factor that activates gene expression during myeloid and B-lymphoid cell development. The nuclear protein binds to a purine-rich sequence known as the PU-box found near the promoters of target genes, and regulates their expression in coordination with other transcription factors and cofactors. The protein can also regulate alternative splicing of target genes. In our transcriptome network, SPI1 appears as a hub which could regulate ICAM-1 which is a gene closely related with lung cancer $(38,39)$.

From the result of regulation network between TFs and pathways in squamous lung cancer, we found that there are many pathways closely related with lung cancer linked by our method. The gene FLI1 is also a hub in our transcriptome network and have a close relationship with lung cancer-related genes including FOS, and ETS2.

The basic understanding of the mechanisms underlying the functioning of lung cancer genes is important for the development of transgenic plants. A deeper understanding of transcription factors and their regulated genes remain an area of intense research activity. Our regulation network is useful in investigating the complex interacting mechanisms of transcription factors and their regulated genes in squamous lung cancer. We also predict that FLI1 and SPI1 are potential candidate genes for squamous lung cancer. However, further experiments are needed to confirm the conclusion.

\section{Acknowledgements}

We wish to express our warm and sincere thanks to Weidong Zang and Lishan Wang from Shanghai Jiaotong University and Fenghe Information and Technology Inc. Their ideas and assistance greatly improved our research and we wish to thank them for all their support.

\section{References}

1. Jemal A, Bray F, Center MM, Ferlay J, Ward E and Forman D: Global cancer statistics. CA Cancer J Clin 61: 69-90, 2011.

2. Travis WD: Pathology of lung cancer. Clin Chest Med 23: 65-81, viii, 2002.

3. Kim HS, Park K, Jun HJ, et al: Comparison of survival in advanced non-small cell lung cancer patients in the pre- and post-gefitinib eras. Oncology 76: 239-246, 2009.

4. Reinmuth N, Mesters RM, Bieker R, Hoffknecht P, Berdel WE and Thomas M: Signal transduction pathways as novel therapy targets in lung cancer. Lung Cancer 45 (Suppl 2): 177-186, 2004.

5. Yagui-Beltran A, He B, Raz D, Kim J and Jablons DM: Novel therapies targeting signaling pathways in lung cancer. Thorac Surg Clin 16: 379-396, vi, 2006. 
6. Daniel VC, Peacock CD and Watkins DN: Developmental signalling pathways in lung cancer. Respirology 11: 234-240, 2006.

7. Denlinger CE, Rundall BK and Jones DR: Modulation of antiapoptotic cell signaling pathways in non-small cell lung cancer: the role of NF-kappaB. Semin Thorac Cardiovasc Surg 16: 28-39, 2004.

8. Stabile LP and Siegfried JM: Estrogen receptor pathways in lung cancer. Curr Oncol Rep 6: 259-267, 2004.

9. Wachi S, Yoneda K and Wu R: Interactome-transcriptome analysis reveals the high centrality of genes differentially expressed in lung cancer tissues. Bioinformatics 21: 4205-4208, 2005.

10. Kanehisa M: The KEGG database. Novartis Found Symp 247: 91-252, 2002

11. Brivanlou AH and Darnell JE Jr: Signal transduction and the control of gene expression. Science 295: 813-818, 2002.

12. Wingender E: The TRANSFAC project as an example of framework technology that supports the analysis of genomic regulation. Brief Bioinform 9: 326-332, 2008.

13. Jiang $C$, Xuan Z, Zhao F and Zhang MQ: TRED: a transcriptional regulatory element database, new entries and other development. Nucleic Acids Res 35: D137-D140, 2007.

14. Smyth GK: Linear models and empirical bayes methods for assessing differential expression in microarray experiments. Stat Appl Genet Mol Biol 3: Article 3, 2004.

15. Maere S, Heymans K and Kuiper M: BiNGO: a Cytoscape plugin to assess overrepresentation of gene ontology categories in biological networks. Bioinformatics 21: 3448-3449, 2005.

16. Shannon P, Markiel A, Ozier O, et al: Cytoscape: a software environment for integrated models of biomolecular interaction networks. Genome Res 13: 2498-2504, 2003.

17. Draghici S, Khatri P, Tarca AL, et al: A systems biology approach for pathway level analysis. Genome Res 17: 1537-1545, 2007.

18. Tavazoie S, Hughes JD, Campbell MJ, Cho RJ and Church GM Systematic determination of genetic network architecture. Nat Genet 22: 281-285, 1999.

19. Draghici S, Khatri P, Martins RP, Ostermeier GC and Krawetz SA: Global functional profiling of gene expression. Genomics 81: 98-104, 2003.

20. Perou CM, Sorlie T, Eisen MB, et al: Molecular portraits of human breast tumours. Nature 406: 747-752, 2000.

21. Vazquez A, Dobrin R, Sergi D, Eckmann JP, Oltvai ZN and Barabasi AL: The topological relationship between the largescale attributes and local interaction patterns of complex networks. Proc Natl Acad Sci USA 101: 17940-17945, 2004.

22. Ideker RE, Huang J, Fast V and Smith WM: Recent fibrillation studies: attempts to wrest order from disorder. Circ Res 89: 1089-1091, 2001.

23. Kitano H, Kataoka K, Furukawa K and Hara S: Specific expression and temperature-dependent expression of the acid protease-encoding gene (pepA) in Aspergillus oryzae in solidstate culture (Rice-Koji). J Biosci Bioeng 93: 563-567, 2002.

24. Keshamouni VG, Reddy RC, Arenberg DA, et al: Peroxisome proliferator-activated receptor-gamma activation inhibits tumor progression in non-small cell lung cancer. Oncogene 23: 100-108, 2004.

25. Reka AK, Goswami MT, Krishnapuram R, Standiford TJ and Keshamouni VG: Molecular cross-regulation between PPARgamma and other signaling pathways: Implications for lung cancer therapy. Lung Cancer 72: 154-159, 2011.
26. Yuan H, Li MY, Ma LT, et al: 15-Lipoxygenases and its metabolites 15(S)-HETE and 13(S)-HODE in the development of non-small cell lung cancer. Thorax 65: 321-326, 2010.

27. Choudhary R, Li H, Winn RA, Sorenson AL, Weiser-Evans MC and Nemenoff RA: Peroxisome proliferator-activated receptorgamma inhibits transformed growth of non-small cell lung cancer cells through selective suppression of Snail. Neoplasia 12: 224-234, 2010

28. Li MY, Yuan H, Ma LT, et al: Roles of peroxisome proliferatoractivated receptor-alpha and -gamma in the development of non-small cell lung cancer. Am J Respir Cell Mol Biol 43: 674-683, 2010

29. Han S, Ritzenthaler JD, Rivera HN and Roman J: Peroxisome proliferator-activated receptor-gamma ligands suppress fibronectin gene expression in human lung carcinoma cells: involvement of both CRE and Sp1. Am J Physiol Lung Cell Mol Physiol 289: L419-L428, 2005.

30. Dergunoa LV, Raevskaia NM, Volosheniuk EL and Limborskaia SA: Expression peculiarities of EGR1, neurotrophins and their receptor genes in human lung cancer and in normal lung tissue. Mol Gen Mikrobiol Virusol 2007: 25-30, 2007 (In Russian).

31. Burcham PC, Raso A and Thompson CA: Toxicity of smoke extracts towards A549 lung cells: role of acrolein and suppression by carbonyl scavengers. Chem Biol Interact 183: 416-424, 2010.

32. Parra E and Ferreira J: The effect of siRNA-Egr-1 and camptothecin on growth and chemosensitivity of breast cancer cell lines. Oncol Rep 23: 1159-1165, 2010

33. Fang L, Min L, Lin Y, et al: Downregulation of stathmin expression is mediated directly by Egr1 and associated with p53 activity in lung cancer cell line A549. Cell Signal 22: 166-173, 2010.

34. Shimoyamada H, Yazawa T, Sato H, et al: Early growth response-1 induces and enhances vascular endothelial growth factor-A expression in lung cancer cells. Am J Pathol 177: 70-83, 2010.

35. Sato Y, Yamamoto N, Kunitoh H, et al: Genome-wide association study on overall survival of advanced non-small cell lung cancer patients treated with carboplatin and paclitaxel. J Thorac Oncol 6: 132-138, 2011

36. Ming J, Zhang Q, Qiu X and Wang E: Interleukin 7/interleukin 7 receptor induce c-Fos/c-Jun-dependent vascular endothelial growth factor-D up-regulation: a mechanism of lymphangiogenesis in lung cancer. Eur J Cancer 45: 866-873, 2009.

37. Zhao Z, Cheng Q, Li X, Wang X and Liu K: c-fos antisense oligodeoxynucleotide reduces VIP-induced upregulation of VEGF expression in small cell lung cancer cells. Zhongguo Fei Ai Za Zhi 9: 312-315, 2006 (In Chinese).

38. Guney N, Soydinc HO, Derin D, et al: Serum levels of intercellular adhesion molecule ICAM-1 and E-selectin in advanced stage non-small cell lung cancer. Med Oncol 25: 194-200, 2008.

39. Dabrowska M, Grubek-Jaworska H, Hoser G, DomagalaKulawik J, Krenke R and Chazan R: Effect of IFN-gamma stimulation on expression of intercellular adhesion molecule-1 (ICAM-1) on alveolar macrophages in patients with non-small cell lung cancer. J Interferon Cytokine Res 26: 190-195, 2006. 